\title{
The Saga of Landau-Gauge Propagators: Gathering New Ammo
}

\author{
Attilio Cucchieri and Tereza Mendes \\ Instituto de Física de São Carlos, Universidade de São Paulo \\ Caixa Postal 369, 13560-970 São Carlos, SP, Brazil
}

\begin{abstract}
Compelling evidence has recently emerged from lattice simulations in favor of the massive solution of the Schwinger-Dyson equations of Landau-gauge QCD. The main objections to these lattice results are based on possible Gribovcopy effects. We recently installed at IFSC-USP a new GPU cluster dedicated to the study of Green's functions. We present here our point of view on the Saga and the status of our project. We also show data for the $2 \mathrm{D}$ case on a $2560^{2}$ lattice.
\end{abstract}

Keywords: Color confinement, Green's functions, Landau gauge, Gribov copies PACS: 12.38.Aw 12.38.Gc 12.38.Lg

\section{THE SAGA}

Intuitively, the explanation of color confinement should be encoded in the infrared (IR) behavior of QCD Green's functions. The Landau-gauge Gribov-Zwanziger (GZ) confinement scenario and the scaling solution obtained by solving Schwinger-Dyson equations (SDEs) demand - for any space-time dimensions $\mathrm{D} \geq 2-$ a null gluon propagator at zero momentum and an IR-enhanced ghost propagator [1]. At present, there is wide agreement [2] that numerical simulations in minimal Landau gauge show (in the infinite-volume limit): 1) an IR-finite gluon propagator $D(p)$ in $\mathrm{D}=3,4$ and a null $D(0)$ in $2 \mathrm{D}, 2)$ violation of reflection positivity for the gluon propagator in $\mathrm{D}=2,3,4$ and 3) an essentially free ghost propagator $G(p)$ in $\mathrm{D}=3,4$ but IR-enhanced in $2 \mathrm{D}$. Thus, the 3D and 4D results support the massive solution of the SDEs $[3,4]$, while the $2 \mathrm{D}$ case has a scaling behavior. Then, a natural question is why the $2 \mathrm{D}$ case is different from the 3D and 4D ones. At the moment, a possible answer to this question has only been presented in [5].

Recently, three works $[6,7,8]$ have allegedly shown evidence of the scaling IR behavior also in 3D and 4D. Here, we will comment on these three works.

\section{The $\beta=0$ Case}

We have already criticized Ref. [6] in our work [9]. Since that criticism has not been answered, we will repeat it here. The authors of Ref. [6] study the Landau-gauge gluon and ghost propagators in the strongcoupling limit of pure SU(2) lattice gauge theory. These propagators are evaluated using different discretizations of the gluon field and, in particular, the standard (com- pact) definition and the (non-compact) stereographic projection [10]. Their main conclusions are: "We furthermore demonstrate that the massive branch observed for $a^{2} q^{2}<1$ does depend on the lattice definition of the gluon fields, and that it is thus not unambiguously defined.... One might still hope that this ambiguity will go away at non-zero $\beta$ in the scaling limit. While this is true at large momenta, we demonstrate...that the ambiguity is still present in the low-momentum region, at least for commonly used values of the lattice coupling such as $\beta=2.3$ or $\beta=2.5$ in $S U(2)$....The scaling properties such as exponent and coupling, on the other hand, appear to be robust under variations of the discretization of the gauge fields...This emphasizes the importance of understanding any discretization ambiguity of the associated gluon mass, before concluding that this mass is now firmly established." However, nowhere in Ref. [6] are data at $\beta=2.5$ shown. On the other hand, data for a lattice volume $32^{4}$ at $\beta=2.5$ in the $\mathrm{SU}(2)$ case are presented in Ref. [10] for the two propagators, using the standard discretization and the stereographic projection. The conclusion of [10] is that "...there are hardly any differences between the propagators obtained in each case". Thus, referring to the last sentence reported above from Ref. [6], there are no discretization ambiguities in the evaluation of these propagators and the existence of a gluon mass is now firmly established.

\section{The Absolute Landau Gauge}

Ref. [7] considers the absolute Landau gauge, i.e. configurations belonging to the fundamental modular region $\Lambda$. This approach, however, cannot yield an IR-enhanced ghost propagator in $3 \mathrm{D}$ or in $4 \mathrm{D}$. Actually, restricting 
the configuration space to the region $\Lambda$ makes the ghost propagator even less singular [11]. This can be seen, indeed, also in Figures 5 and 12 of Ref. [7]. The author tries to explain these results, which clearly go against the scaling solution, by saying that "The reason for this behavior of the ghost propagator...may be connected to the volume evolution of the first Gribov region and the fundamental modular region....The combined effect of the precise shape of the low-eigenvalue spectrum and a diverging normalization of the eigenstates could be sufficient to provide a more infrared divergent ghost propagator in the infinite-volume and continuum limits in absolute Landau gauge than in minimal Landau gauge.” Thus, simulations in the absolute Landau gauge should agree with the scaling solution in the infinite-volume limit (for $\mathrm{D}=3,4)$ due to a hypothetical diverging contribution of the eigenstates of the Faddeev-Popov operator. (Note that a possible way of quantifying this sentence would be to prove that the lower bound of the ghost propagator, introduced in Ref. [12], blows up sufficiently fast in the infinite-volume limit.) Moreover, that this effect should be important in the absolute Landau gauge and not in the minimal Landau gauge remains a mystery to us, considering that any configuration belonging to the absolute Landau gauge is also a configuration of the minimal Landau gauge. The author also adds "A final proof is, of course, only that in the absolute Landau gauge at sufficiently large volume the ghost propagator would be more singular than in the minimal Landau gauge. The volume dependence of the propagator in both gauges found here is as expected if this is the case....Hence, at the current point it seems more appropriate to compare the lattice results in absolute Landau gauge, rather than in minimal Landau gauge, with those from functional results which exhibit a scaling behavior in the far infrared." These statements - which are somewhat sibylline, since the data do not show an IR-enhanced ghost propagator - may be the reason why Ref. [7] is (wrongly) cited as a numerical verification of the scaling behavior for $\mathrm{D}$ $>2$.

\section{The 2D Case}

Let us note that if the massive behavior observed in 3D and in 4D could be related to discretization effects, as suggested by Ref. [6], or to Gribov-copy effects, as reported in [7], then these effects should also be present for $\mathrm{D}=2$ and one should not find in this case a scaling behavior. In this respect, still in Ref. [7], the author makes the following prediction: "A consequence of this scenario is that it should be expected that also in two dimensions, for sufficiently large volumes and number of Gribov copies, an infrared finite gluon propagator is obtained in the minimal Landau gauge." We checked this

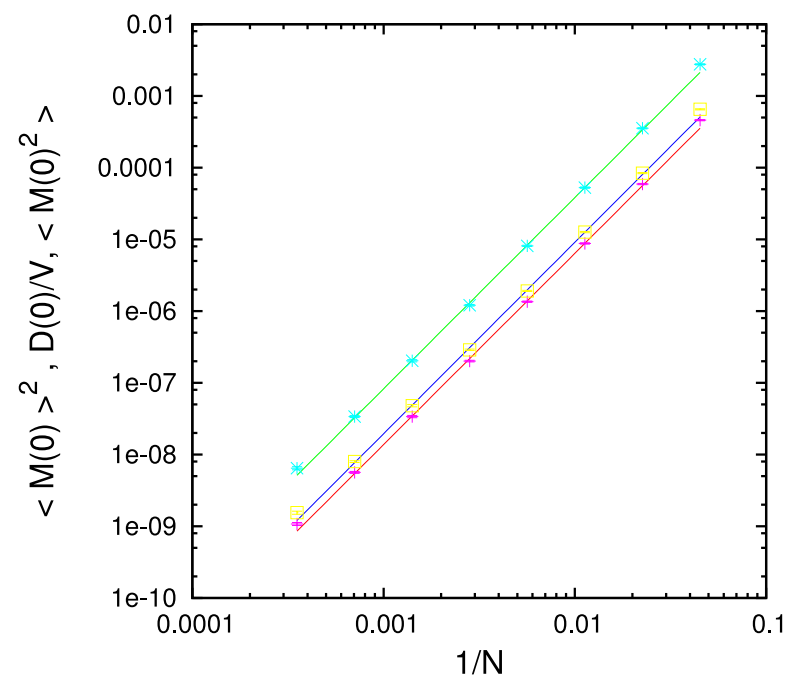

FIGURE 1. Plot of $D(0) / V$, together with its upper and lower bounds [13], as a function of the inverse lattice side $1 / N$. In the three cases we get a behavior $1 / N^{e}$ with $e=2.67(2)$.

prediction by evaluating the gluon propagator for lattice volumes up to $2560^{2}$ at $\beta=10$ (i.e. with a lattice size $L \approx 460 \mathrm{fm}$ ). In Fig. 1 we plot the volume dependence of $D(0) / V$, together with the upper and lower bounds introduced in Ref. [13]. The three sets of data clearly extrapolate to zero faster than $1 / V$, implying $D(0)=0$ in $2 \mathrm{D}$.

\section{The B Gauges}

After considering the absolute Landau gauge in Ref. [7], a new set of gauges - called B gauges - was introduced by the same author [8]. In this case one looks along each orbit for a transverse configuration that yields a given value $B$ for the ghost dressing function $D_{G}(p)=$ $p^{2} G(p)$ at the smallest non-zero momentum $p_{\min }$. This definition does not solve the Gribov ambiguity [8]. Moreover, in order to find an IR-enhanced ghost propagator one needs to favor configurations closer to the first Gribov horizon $\partial \Omega$. This is the opposite of what is done in the absolute Landau gauge, where one favors configurations well inside the first Gribov region $\Omega$ [14]. Thus, if the B gauges should produce the scaling solution, "it could well be that...the absolute Landau gauge is not connected to a scaling behavior" [8], in disagreement with Ref. [7].

The main result of this approach is that the ghost propagator is strongly affected by the choice of configuration on each orbit, in such a way that its values are enclosed in a "corridor". In particular, in 3D and 4D the upper bound of this corridor "is strongly increasing with volume". At the same time, the gluon propagator seems 
to be $B$-independent and we should have $D(0)>0$ in the infinite-volume limit. Thus, the only scaling solution that can be obtained with the $\mathrm{B}$ gauges seems to be the one corresponding to a critical exponent $\kappa=1 / 2$, which was never the preferred value in scaling-solution works. Moreover, if the infrared exponent is $1 / 2$ then in $4 \mathrm{D}$ one should have $D_{G}(p) \sim 1 / p$. Since $1 / p_{\text {min }} \approx L$, the upper bound of the corridor should grow at least as fast as the lattice size $L$, in order to support the scaling solution. One can verify that this is not the case with the 4D data presented in Fig. 3 of [8] (the curve should be hyperbolic as a function of $1 / L)$.

Let us note that one of the motivations for the introduction of the B gauges is the possible relation with the one-parameter family of solutions obtained by functional methods $[3,15]$. In this respect one should stress, however, that the B gauges are related to different Gribov copies on each orbit. On the other hand, the configuration space is not encoded in the SDEs and this information has to be put in by hand. This can be done in simple cases [16], if all Gribov copies are known, but nobody knows how to do it in a realistic case. Thus, this relation seems at the moment quite accidental. Even more fanciful seems to us the hypothetical connection between the Kugo-Ojima (KO) approach [1] and a (possible) scaling solution obtained using B gauges. Indeed, this connection requires "subtle cancellation" [8], since one has to relate an average over all Gribov copies to results obtained by selecting specific copies inside the first Gribov region $\Omega$. In our opinion, the lack of BRST invariance when the functional space is restricted to $\Omega$ [17] obscures the relation of the GZ approach with the $\mathrm{KO}$ criterion and the analogies between these two approaches seem to be, at the moment, a questionable coincidence.

Finally, several questions should be answered before discussing in detail the results obtained using B gauges. For example, it is well known that some Gribov copies on the lattice are just lattice artifacts. Thus, by using the B gauges, aren't we just probing these artifacts? This may explain the over-scaling observed in [18]. Also, it seems very difficult to control the infinite-volume limit of the corridor and, as pointed in [8], "it cannot be excluded that the corridor closes again at much larger volumes". This seems indeed possible since, for very large lattice volumes, all the orbits should come very close to the boundary $\partial \Omega$ and one can expect smaller Gribov-copy effects [19].

\section{CONCLUSIONS: THE GHOST FACTORY}

We believe that, in order to understand the results obtained in minimal Landau gauge using numerical simulations, the first question to be answered is: why is the $2 \mathrm{D}$ case different? One could also ask: can we test nu- merically the explanation presented in [5]? A clear answer to these questions probably requires new ideas and better data (especially in the ghost sector). Unproven hypotheses and happy coincidences should on the contrary be treated with great caution.

We recently installed at IFSC-USP a new machine with 18 CPUs Intel quadcore Xeon $2.40 \mathrm{GHz}$ (with InfiniBand network and a total of 216 GB of memory) and 8 NVIDIA Tesla S1070 boards (500 Series), each with 960 cores and 16 GB of memory. The peak performance of the 8 Tesla boards is estimated in about 2.8 Tflops in double precision and 33 Tflops in single precision. This machine will be used mainly for studies of Green's functions in different gauges (Landau, Feynman and Coulomb) for various $\mathrm{SU}\left(N_{c}\right)$ gauge groups. In particular, the GPUs will be used for the inversion of the Faddeev-Popov matrix using conjugate gradient. This computer will allow us to perform an extensive study of the ghost sector. We believe that this new ammo will help us clarify the issues addressed above.

\section{ACKNOWLEDGMENTS}

We thank FAPESP (grant \# 2009/52213-2) and CNPq for support.

\section{REFERENCES}

1. For a concise discussion see A. Cucchieri, T. Mendes, [arXiv:0809.2777 [hep-lat]] and references therein.

2. For a review see A. Cucchieri, T. Mendes, PoS QCDTNT09, 026 (2009).

3. P. Boucaud et al., JHEP 0806, 012 (2008).

4. J. Papavassiliou, Nucl. Phys. Proc. Suppl. 199, 44-53 (2010); A. C. Aguilar, D. Binosi, J. Papavassiliou, Phys. Rev. D81, 125025 (2010).

5. D. Dudal et al., Phys. Lett. B680, 377-383 (2009).

6. A. Sternbeck, L. von Smekal, Eur. Phys. J. C68, 487-503 (2010).

7. A. Maas, Phys. Rev. D79, 014505 (2009).

8. A. Maas, Phys. Lett. B689, 107-111 (2010).

9. A. Cucchieri, T. Mendes, Phys. Rev. D81, 016005 (2010).

10. L. von Smekal et al., PoS LAT2007, 382 (2007).

11. A. Cucchieri, Nucl. Phys. B508, 353-370 (1997).

12. A. Cucchieri, T. Mendes, Phys. Rev. D78, 094503 (2008).

13. A. Cucchieri, T. Mendes, Phys. Rev. Lett. 100, 241601 (2008).

14. A. Cucchieri, Nucl. Phys. B521, 365-379 (1998).

15. C. S. Fischer, A. Maas, J. M. Pawlowski, Annals Phys. 324, 2408-2437 (2009).

16. H. Reinhardt, W. Schleifenbaum, Annals Phys. 324, 735-786 (2009).

17. D. Dudal et al., Phys. Rev. D 79 (2009) 121701.

18. A. Maas et al., Eur. Phys. J. C68, 183-195 (2010).

19. A. Sternbeck et al., Phys. Rev. D72, 014507 (2005). 